\title{
Planetary system and star formation science with non-redundant masking on JWST
}

\author{
Anand Sivaramakrishnan ${ }^{a}$, Peter G. Tuthill ${ }^{b}$, Michael J. Ireland ${ }^{b}$, \\ James P. Lloyd ${ }^{c}$, Frantz Martinache ${ }^{d}$, Rémi Soummer ${ }^{e}$, Russell B. Makidon ${ }^{e}$, \\ René Doyon ${ }^{f}$, Mathilde Beaulieu ${ }^{f}$, and Charles A. Beichman ${ }^{g}$ \\ ${ }^{a}$ Department of Astrophysics, American Museum of Natural History, \\ 79th Street at CPW, New York, NY 10024 United States \\ ${ }^{b}$ School of Physics, University of Sydney, NSW Australia \\ ${ }^{c} 230$ Space Sciences Building, Cornell University, Ithaca, NY 14853 United States ${ }^{d}$ Subaru \\ Telescope, 650 N. A'ohoku Place, Hilo, HI 96720, United States \\ ${ }^{e}$ Space Telescope Science Institute, 3700 San Martin Drive, \\ Baltimore, MD 21218, United States; \\ ${ }^{f}$ Département de Physique, Université de Montréal, Montréal, Qc, Canada \\ ${ }^{g}$ NASA ExoPlanet Science Institute, California Institute of Technology, \\ 1201 California Blvd., Pasadena CA 91125, United States
}

\begin{abstract}
Non-redundant masking (NRM) is a high contrast high resolution technique that is relevant for future space missions dedicated to either general astrophysics or extrasolar planetary astronomy. NRM mitigates not only atmospheric but instrument-induced speckle noise as well. The recently added mask in the Fine Guidance Sensor Tunable Filter Imager (FGS-TFI) on the James Webb Space Telescope (JWST) will open up a search space between 50 and 400 mas at wavelengths longer than $3.8 \mu \mathrm{m}$. Contrast of $10^{4}$ will be achievable in a 10 ks exposure of an $M=7$ star, with routine observing, target acquisition, and data calibration methods. NRM places protoplanets in Taurus as well as Jovians younger than $300 \mathrm{Myr}$ and more massive than $2 M_{J}$ orbiting solar type stars within JWST's reach. Stars as bright as $M=3$ will also be observable, thus meshing well with next-generation ground-based extreme adaptive optics coronagraphs. This parameter space is inaccessible to both JWST coronagraphs and future 30-m class ground-based telescopes, especially in the mid-IR. We show that NRM used on future space telescopes can deliver unsurpassed image contrast in key niches, while reducing mission risk associated with active primary mirrors.
\end{abstract}

Keywords: Extrasolar planets, JWST, PSF subtraction, high-contrast imaging

\section{INTRODUCTION}

Landmark discoveries such as dusty disks imaged around young stellar objects, mass-loss shells of evolved stars and the fascinating time-varying spiral plumes surrounding dusty Wolf-Rayet systems have been reported amongst the 50-odd peer-reviewed papers describing results produced by this technique (Ref. 1-15). However, the atmosphere places limitations on non-redundant masking (NRM) performance.

NRM on a space telescope has not been described in the literature to date. We present detailed simulations of NRM performance on the James Webb Space Telescope (JWST) to demonstrate the exciting planetary science enabled by the recent addition of NRM to JWST's suite of established instruments, and to emphasize the importance of this technique to other future space-based observatories. On JWST NRM will widen the telescope's science reach to include a unique combination of wavelength and angular resolution regimes inaccessible from the ground, even with the advent of extreme adaptive optics (ExAO) and coronagraphs behind $30 \mathrm{~m}$ extremely

Further author information: (Send correspondence to A.S. E-mail: anand@amnh.org

Techniques and Instrumentation for Detection of Exoplanets IV, edited by Stuart B. Shaklan, Proc. of SPIE Vol. 7440, 74400Y · @ 2009 SPIE · CCC code: 0277-786X/09/\$18 · doi: 10.1117/12.826633 


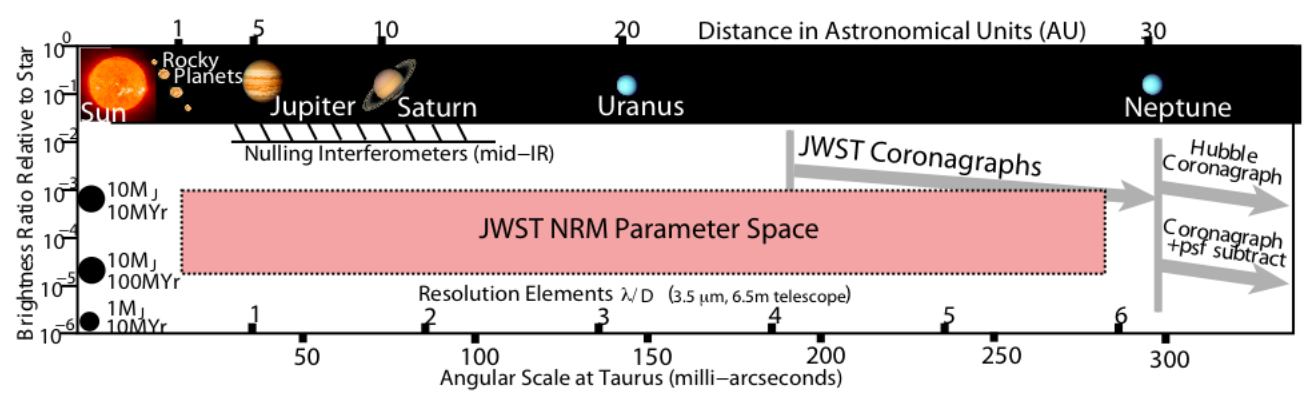

Figure 1. The parameter space available to the newly-added non-redundant sparse aperture mask on JWST's FGS-TFI instrument. It is designed to operate at 3.8 microns and longer.

large telescopes of the future. For example, NRM used at $4 \mu \mathrm{m}$ will place warm extrasolar jovians within 4 to 30 AU of F, G, and K dwarfs 30pc from the Sun within JWST's purview (Figure 1).

Indeed, NRM can add high resolution capability to large filled-aperture space telescopes without sacrificing their wide utility for general astrophysical observations. Furthermore, the NRM search space is complementary to future coronagraphic space missions dedicated to extrasolar planet imaging and characterization, as well as to ground-based ExAO instruments, ${ }^{16,17}$ in that it yields moderate contrasts between 0.5 and $4 \lambda / D$ ( $\lambda$ being the observing wavelength, $D$ the telescope diameter). Diffraction-limited stellar coronagraphy, on the other hand, typically covers a search space at higher contrast, at separations larger than $\sim 4 \lambda / D$ (Ref. 18). Techniques such as Angular or Spectral Differential Imaging (e.g., Ref. 19,20 and references therein) do not work well at these small angular separations. In addition, NRM data has a high degree of self-consistency that is absent in full-aperture direct or coronagraphic imaging.

We examine the scientific merit and feasibility of NRM on JWST's Fine Guidance Sensor's Tunable Filter Imager (FGS-TFI) ${ }^{21}$ between $3.8 \mu \mathrm{m}$ and $5 \mu \mathrm{m}$, operating at a spectral resolution of 100 . These contrast predictions are relevant to JWST science planning for its newly-added high angular resolution capability. Our NRM designs work well in 10\% bandpass filters, making NRM a possible technique for JWST's near-IR imager NIRCam and mid-IR imager MIRI. ${ }^{22}$ Our results can be scaled with both wavelength and optical path difference stability when planning NRM on future missions.

JWST's target acquisition methods, pointing stability, and data calibration pipeline processing are aimed at general purpose imaging with an undemanding operating protocol. They will suffice for the high dynamic range NRM observations we describe here. In contrast, coronagraphic observations require specialized target acquisition and peak-up, ${ }^{23}$ very small pointing errors, ${ }^{24}$ exquisite wavefront flatness and highly uniform pupil illumination. In comparison, NRM relies on long-term stability of the telescope's wavefront during a several minute or hour long exposure, rather than on any stringent requirements on wavefront quality. It therefore mitigates some of the risk associated with the complex mechanisms and sequences involved in co-phasing JWST's 18-segment, deployable $6.5 \mathrm{~m}$ primary mirror (PM) as the telescope orbits the second Earth-Sun Lagrangian equilibrium point (e.g., Ref. 25).

\section{NRM INTERFEROMETRY AND ITS LIMITS}

Interferometry with non-redundant baselines was developed for radio astronomy, ${ }^{26}$ and subsequently adapted to optical wavelengths. ${ }^{27}$ Today it is used in IR and optical bandpasses, often behind instruments not originally developed for sparse aperture interferometry. ${ }^{1}$

Refs. 4,28-30 and references therein present a fuller description of NRM. In brief, a non-redundant array of subapertures is achieved with an $N$-holes $\left(\left\{h_{1}, h_{2}, \ldots, h_{N}\right\}\right)$ placed so no vector (baseline) between the centers of two holes is repeated (see Figure 2, left). The resulting PSF is created by several coherent fringe patterns (each with different angular periods on the detector, thanks to non-redundancy) overlaid across one another. A fringe formed by holes $h_{i}$ and $h_{j}$ is quantified by a complex visibility, which has an amplitude (degree of modulation) and a phase (the offset of the fringe's center from the centroid of the PSF). The fringe visibility is the complex 

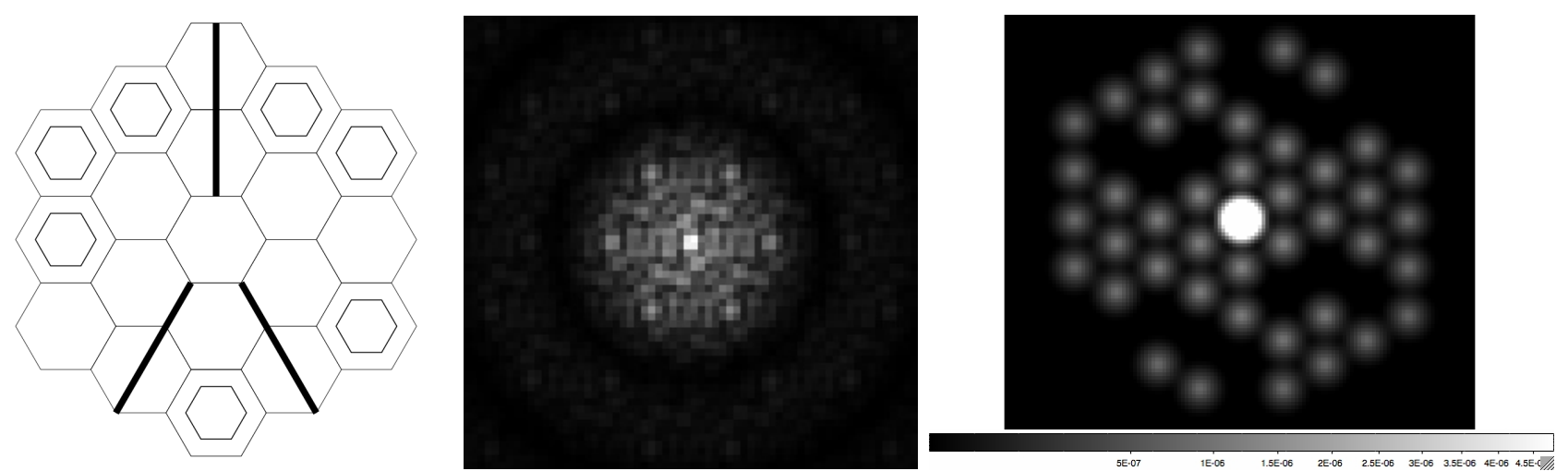

Figure 2. Left: The FGS-TFI 7-hole non-redundant pupil mask (holes) overlaid on the JWST primary mirror. The secondary mirror supports are shown as three thick black lines. The mask has undersized holes to mitigate against pupil shear. Center: A $4.05 \mu \mathrm{m}$ FGS-TFI image of a point source, with a $1 \%$ bandwidth filter, on a square root logarithmic stretch. The pixel scale (65 mas) is that of FGS-TFI. The first Airy ring diameter is $2.4^{\prime \prime}$. Generally data out to the first Airy ring is analyzed. Right: the power spectrum of the image (also on a square root scale), showing fringe power on the 21 baselines passed by the mask.

product of a Fourier component of the object and a system visibility. When observing a point-source, this phase $\phi_{i j}$ measures the piston wavefront error between the two holes. A closure phase is formed by the addition of three fringe phases created by a triangle of holes, $\left\{h_{i}, h_{j}, h_{k}\right\}$, notably $\phi_{i j}+\phi_{j k}+\phi_{k i}$. Although piston wavefront errors change the fringe phases, the closure phases are insensitive to these wavefront errors and only measure source structure. There are $N(N-1) / 2$ independent closure phases measurable in the image.

NRM is a form of imaging with a PSF that looks unusual. The NRM PSF displays multiple sharp peaks, whereas traditional diffraction-limited PSFs are dominated by one peak (Figure 2, center). However, the NRM PSF possesses some useful properties. First, through the use of closure phase, it is insensitive to large scale wavefront errors over the pupil. Second, its core is more than twice as narrow as a traditional PSF's (the Michelson criterion, $0.5 \lambda / D$, cf. Rayleigh's criterion, $1.22 \lambda / D$ ). Third, NRM data sets produce results that can be averaged to reduce noise, even in the presence of slowly-varying speckles. By comparison, co-adding PSFs in the presence of speckle noise is always limited by a speckle noise floor. ${ }^{31-33}$

On the ground NRM filters out aberrations that pass through an AO system. It is insensitive to the noncommon path (NCP) aberrations between the science and sensing arms of the instrument. The NCP issue has only recently been addressed by specialized calibration systems in future ExAO coronagraphic systems utilizing full-aperture pupils. ${ }^{34}$ Today's ground-based NRM routinely achieves stability of 0.5 degrees on the closure phase, hence passively stabilizing the phase at the level of $\lambda / 500$ to $\lambda / 1000$.

For a point source any closure phase must be zero. A measured non-zero closure phase in the data provides spatial information on the object. A consistent, systematic, instrument-induced departure from a zero closure phase can be measured using a calibration star, and subtracted from a target's closure phase. Conceptually, closure phase data analysis is a model fit of the intensity distribution on sky, the observations being closure phases from all the closed triangles that can be constructed with the given set of baselines. Fringe visibility data makes solving for the actual image (or its measured Fourier components) possible. The inner working angle (IWA) of such an image is $0.5 \lambda / D$, the outer working angle (OWA) is set by the shortest non-redundant baseline available. Recovering the measured Fourier components of source structure with NRM imaging using both closure phases and fringe visibilities is a well-posed problem. This is not the case with full aperture imaging. Ground-based fringe visibilities suffer from temporal instabilities due to atmospheric scintillation and transparency variations, though closure phases still extremely useful. On JWST fringe visibilities should be stable since segment reflectivity is unlikely to vary perceptibly during a single exposures, or between science and calibrator exposures.

In practice dynamic range may be limited by effects such as a non-isotropic guiding error, detector flat field errors, and pupil wander. Target placement repeatable to an arcsecond on JWST's HAWAII-2RG detectors will 


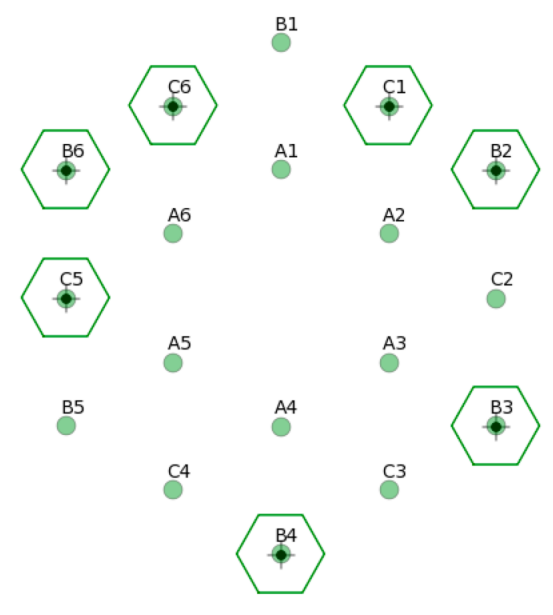

Figure 3. The pupil plane in FGS-TFI is decentered, demagnified, stretched slightly in one dimension, and slightly rotated relative to JWST's primary mirror (PM). The NRM in the JWST FGS-TFI pupil wheel is shown in PM coordinates, after being projected back to the PM using JWST's optical design. Each of the 18 segment centers are indicated with light green circles labelled with their segment names (A1,A2,.., C6). The conceptual design NRM with holes placed in a regular hexagonal grid in (V2,V3) space is shown by small black solid circles at the nominal segment centers' locations (i.e., assuming segments form a regular hexagonal grid in (V2,V3) space). The projected mask's holes in PM space are the 7 green hexagons. Slight misalignments of the holes with their respective PM segment centers will not affect the scientific capabilities of the NRM. However it is preferable that the NRM's holes do not span a segment-to-segment discontinuity. Undersizing of the mask's holes relative to the segments themselves allows for pupil shear up to $3.8 \%$ of the pupil diameter before the mask's holes cross segment boundaries.

improve data calibration, given the spatial frequency of the detector' flat field structure.

\section{NRM IN SPACE}

Without AO, ground-based NRM, like speckle interferometry, must freeze temporally-varying fringe patterns. AO stabilizes relative piston differences between subapertures in the aperture mask. This enables longer exposure times, limited by pixel well-depth, thermal background rates or instrument stability. Once deployed, JWST's primary mirror segments' positions relative to each other are expected to drift very slowly. Exposure times will likely be limited by cosmic rays, with a maximum single exposure time measured in hours. Under such conditions, NRM opens up a search space completely beyond the reach of ground-based telescopes. Thermal background and atmospheric opacity also limit ground-based imaging longward of about $2 \mu \mathrm{m}$. Furthermore, JWST's unusual primary mirror geometry and articulation are unlikely to cause problems for NRM interferometry.

For a $0.5 \mathrm{~m}^{2}$ subaperture at $4 \mu \mathrm{m}$, a $1 \%$ filter bandwidth and $50 \%$ net quantum efficiency, $M \simeq 7$ is the limit for $10^{4}$ contrast in $10 \mathrm{ks}$, and $M=12$ the limit for $10^{3}$ contrast in 10ks. A $10 \%$ bandwidth filter will reduce exposure times by a factor of ten for the same contrast. At bandwidths in excess of $10 \%$ the 7 -hole FGS-TFI designs' contrast starts to degrade. In FGS-TFI, sub-array readouts will enable observations of objects as bright as $M=3$.

\section{SIMULATIONS}

We used ten Monte Carlo realizations of JWST's exit pupil wavefront that conform to JWST's wavefront error budget. ${ }^{39}$ These OPDs ${ }^{40}$ provided with the JWPSF $^{41}$ software distribution, represent the wavefront in JWST NIRCam's short-wavelength channel. They assume particular low-, mid-, and high-spatial frequency wavefront 


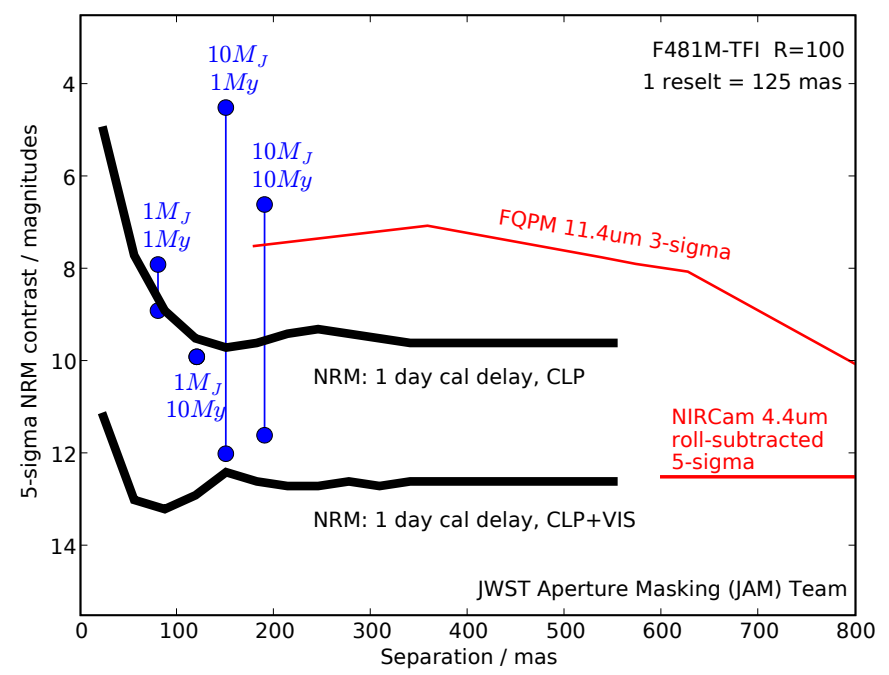

Figure 4. Predicted JWST NRM and coronagraph performance. Dynamic range using only closure phase (CLP) as well as closure phase in combination with fringe visibility (CLP+VIS) data for $1 \%$ bandpass NRM imaging at $4.81 \mu \mathrm{m}$ (using the 7-hole mask shown in Figure 1) in JWST's FGS Tunable Filter Imager (TFI) is plotted (heavy curves). A 1 day delay between the NRM target and calibrator stars, with worst-case attitude-dependent thermally induced primary mirror configuration changes (breathing), guiding errors, and measured detector intra-pixel sensitivity are simulated. In a 10 ks exposure on an $M=7$ target, photon noise sets a 10 magnitude dynamic range limit. $10 \%$ bandwidths will produce similar results in $1 \mathrm{ks}$. The range of estimated contrasts between a solar type star and 1 and 10 Jupiter mass planets, at ages of 1 and 10 Myr (Ref. 35,36) are shown (vertical blue lines placed at arbitrary separations). This mask will enable JWST to detect protoplanets in Taurus. The same mask in JWST NIRCam will produce 1 to 2 magnitudes less contrast at $2 \mu \mathrm{m}$, at half the corresponding angular separation. In MIRI NRM can produce even higher contrast than in FGS-TFI. NRM performance at $4.8 \mu \mathrm{m}$ drops outside about 600mas. NIRCam's linear band-limited coronagraph performance using planned JWST roll angles ${ }^{37}$ complements the FGS-TFI NRM search space. The JWST MIRI four quadrant phase mask coronagraph $11 \mu \mathrm{m}$ contrast curve, ${ }^{38}$ assuming a static $5 \mathrm{mas}$ pointing error between target and calibrator, no guiding error, unrestricted telescope roll capability, and no error in calibration star placement is also plotted. Jovian planets are brighter at this wavelength than at 3-5 $\mu \mathrm{m}$. 


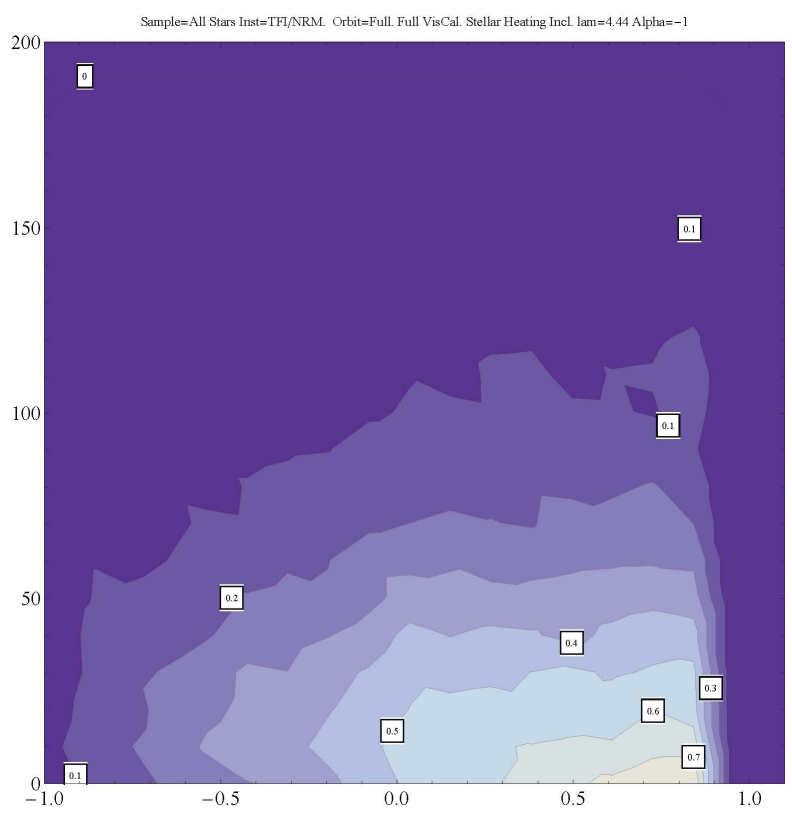

Figure 5. NRM detection probabilities for FGS-TFI at $4.44 \mu \mathrm{m}$, drawing from a large sample of young nearby stars. Companion mass is cut off above 10 Jupiter masses. Contours show the estimated probability of finding a planet with a given mass (in Jupiter masses, log scale), on the horizontal axis, and a given semi-major axis (AU, vertical axis) within nominal performance (a 10 magnitude contrast floor imposed on the curves in Fig. 4). The exposure time assumed is about 1 hour per source. Better detection probabilities would result from longer exposures on an individual object.

error allocations to JWST's Optical Telescope Element (OTE) and Integrated Science Instrument Module (ISIM). These realizations yield PSFs at $2 \mu \mathrm{m}$ with Strehl ratios above $80 \%$. They are estimates, given the expected residual wavefront error 14 days after active tuning of the JWST primary mirror (PM) figure has been performed, and the telescope slewed from a spacecraft attitude with the most thermal load from the Sun to one with the least such thermal load. To preserve the individual segment wavefront errors associated with one realization of the 18 segments in JWST's PM, we extracted the individual segment piston errors from each of the ten wavefront maps, and used them to create ten instances of a single thermally perturbed set of PM segments.

Using methods described in Refs. 42-45, we simulated images on a 9-fold finer grid than the 65 mas FGS-TFI detector pixel pitch. We dithered the oversampled PSFs by one sample spacing to model pointing errors of $7 \mathrm{mas}$ per axis. Intra-pixel quantum efficiency was modeled as a quadratic, ranging from unity at a pixel center to 0.8 at a pixel corner. We then binned the $9 \times 9$ dithered subsampled PSFs to create an image on the FGS-TFI pixel scale. We did not explicitly simulate photon noise, read out noise $\left(\sim 15 e^{-} \mathrm{rms}\right.$ for a double-correlated sample), or detector flat field errors, although our detection threshold calculations have taken some experience-based account of these processes.

Simulated data were analyzed with a pipeline used for Keck, Palomar, and VLT data. (e.g., Ref. 46). Nine of the ten PSF realizations in a set were taken to be calibrator observations, and one of the ten was taken to be the target observation. This process was repeated for several choices of target PSF. Rather than replicating the complex Monte-Carlo process of Ref. 46, we chose to use a simple detection threshold of $5 \sigma$, with closure-phase and visibility standard deviations calculated from scatter within the 9 calibrator observations. Note that typical 99.9\% detection thresholds in Ref. 46 were $\sim 4$ to $4.5 \sigma$ with a more complete Monte-Carlo, which was verified by the lack of numerous false detections in that paper. We therefore believe our $5 \sigma$ limit is conservative.

Figure 4 shows the contrast achieved with dynamic range calculated two ways: first, using only the closure phases (labelled CLP) for a comparison with current ground-based results, and second, using both closure phases and fringe visibilities (labelled CLP+VIS), to estimate the full range of contrast available to JWST. Contrast between $M_{J}$ and $10 M_{J}$ planets 1 and $10 \mathrm{Myr}$ old and a parent G2V dwarf are shown in the figure. We find our mask designs produce these estimated contrasts even when the detector's pixel pitch is slightly worse than 


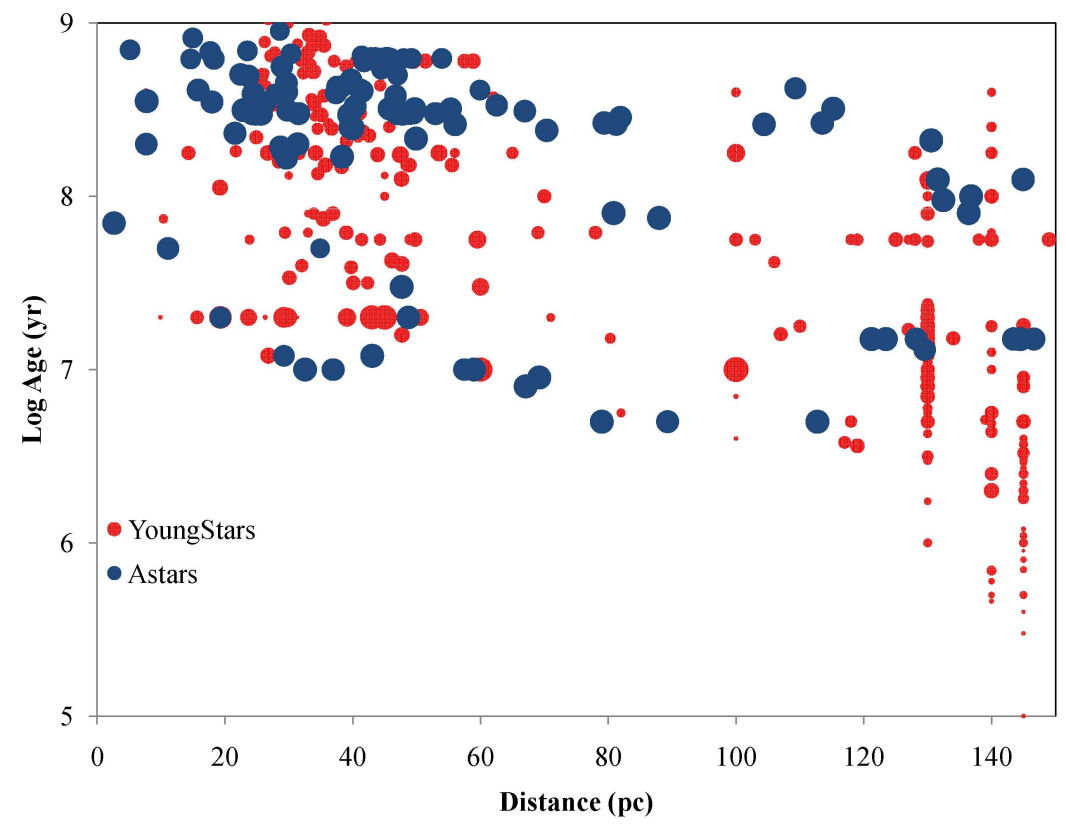

Figure 6. Distribution of stellar ages with distance in the sample used to generate the detection probability shown in Fig. 5. The sample comprises stars younger than $5 \mathrm{Myr}$ and closer than $140 \mathrm{pc}$ from the Sun, and stars older than $10 \mathrm{Myr}$ that are within $50 \mathrm{pc}$ of the Sun.

Nyquist. The very highest contrast shown is obtained using both closure phases as well as visibilities.

Dithering and drizzling images ${ }^{47}$ may further enable undersampled data recovery. Information from routine wavefront sensing observations ${ }^{45}$ may help improve the dynamic range of NRM data. We have not examined these two refinements.

\section{BENEFITS OF NRM IN SPACE}

NRM brings both scientific and operational advantages to JWST, given the telescope's segmented architecture and planned co-phasing methods during commissioning. ${ }^{48-50}$ Every NRM image measures the relative piston between each hole in the aperture mask, with a capture range set by the filter coherence length. Images taken through two masks with $\sim 5 \%$ throughput can measure segment pistons and tilts to interferometric precision, without the need for any alteration of the optical train, whether they be defocus, segment tilt, or indeed any other active mirror motion. Coarse co-phasing JWST can be accomplished using $1 \%$ bandpass filters and NRMs. Masks in separate cameras mitigate instrument failure-induced risk, and enables campaign mode observing with the best image quality possible in any camera equipped with two NRM's. Two such masks in a camera can measure its field-dependent and chromatic aberrations. Camera-specific wavefront knowledge can feed into data reduction, benefiting science that requires thorough understanding of temporal, spatial, and chromatic variations in the PSF. In addition, NRM observations determine stellar multiplicity at the highest resolution and contrast possible. Such observations can eliminate inappropriate wavefront sensing or guide star choices. Images taken as the mask is stepped across the pupil (or vice versa) will measure pupil location without specialized pupil imaging optics. Such information is relevant for IR instrument and telescope maintenance. 


\section{CONCLUSION}

Detailed simulations with time-varying mirror figure errors and existing data reduction methods suggest that placing a non-redundant aperture mask on any of the JWST instruments brings exciting high resolution high contrast imaging within reach. The 7-hole NRM in the Fine Guidance Sensor's Tunable Filter Imager will image protoplanets in Taurus (see Figure 5 and 6). Such aperture masking provides JWST with alternative, risk-reducing wavefront measuring techniques, while increasing the science output of JWST substantially, and paving the way for future missions with NRM instrumentation. Future missions can increase the science payoff with more optimized NRM modes, making the technique interesting for galactic, extragalactic, and cosmological observations.

\section{ACKNOWLEDGMENTS}

We thank P. A. Lightsey, J. C. Mather, M. Robberto and N. Rowlands for encouraging discussions and helpful information. This work is supported in part by the National Science Foundation under Grant AST 08-04417.

\section{REFERENCES}

[1] Tuthill, P. G., Monnier, J. D., Danchi, W. C., and Haniff, C. A., "Michelson interferometry with Keck I," in [SPIE Conference Series], Reasenberg, R. D., ed., 3350, 839-846 (July 1998).

[2] Monnier, J. D., Tuthill, P. G., and Danchi, W. C., "Pinwheel Nebula around WR 98A," ApJL 525, L97-L100 (Nov. 1999).

[3] Monnier, J. D., Tuthill, P. G., Lopez, B., Cruzalebes, P., Danchi, W. C., and Haniff, C. A., "The Last Gasps of VY Canis Majoris: Aperture Synthesis and Adaptive Optics Imagery," ApJ 512, 351-361 (Feb. 1999).

[4] Tuthill, P. G., Monnier, J. D., Danchi, W. C., Wishnow, E. H., and Haniff, C. A., "Michelson Interferometry with the Keck I Telescope," PASP 112, 555-565 (Apr. 2000).

[5] Tuthill, P. G., Monnier, J. D., and Danchi, W. C., "A dusty torus around the luminous young star LkH $\alpha 101$," Nature 409, 1012-1014 (Feb. 2001).

[6] Tuthill, P. G., Monnier, J. D., Danchi, W. C., Hale, D. D. S., and Townes, C. H., "Imaging the Disk around the Luminous Young Star LkH $\alpha 101$ with Infrared Interferometry," ApJ 577, 826-838 (Oct. 2002).

[7] Tuthill, P. G., Monnier, J. D., and Danchi, W. C., "Multiwavelength Diffraction-limited Imaging of the Evolved Carbon Star IRC +10216. II.," ApJ 624, 352-358 (May 2005).

[8] Tuthill, P., Monnier, J., Tanner, A., Figer, D., Ghez, A., and Danchi, W., "Pinwheels in the Quintuplet Cluster," Science 313, 935-+ (Aug. 2006).

[9] Lloyd, J. P., Martinache, F., Ireland, M. J., Monnier, J. D., Pravdo, S. H., Shaklan, S. B., and Tuthill, P. G., "Direct Detection of the Brown Dwarf GJ 802B with Adaptive Optics Masking Interferometry," ApJL 650, L131-L134 (Oct. 2006).

[10] Pravdo, S. H., Shaklan, S. B., Wiktorowicz, S. J., Kulkarni, S., Lloyd, J. P., Martinache, F., Tuthill, P. G., and Ireland, M. J., "Masses of Astrometrically Discovered and Imaged Binaries: G78-28AB and GJ 231.1BC," ApJ 649, 389-398 (Sept. 2006).

[11] Martinache, F., Lloyd, J. P., Ireland, M. J., Yamada, R. S., and Tuthill, P. G., "Precision Masses of the Low-Mass Binary System GJ 623," ApJ 661, 496-501 (May 2007).

[12] Tuthill, P. G. and Lloyd, J. P., "A Symmetric Bipolar Nebula Around MWC 922," Science 316, 247- (Apr. 2007).

[13] Ireland, M. J. and Kraus, A. L., "The Disk Around CoKu Tauri/4: Circumbinary, Not Transitional," ApJL 678, L59-L62 (May 2008).

[14] Ireland, M. J., Kraus, A., Martinache, F., Lloyd, J. P., and Tuthill, P. G., "Dynamical Mass of GJ 802B: A Brown Dwarf in a Triple System," ApJ 678, 463-471 (May 2008).

[15] Tuthill, P. G., Monnier, J. D., Lawrance, N., Danchi, W. C., Owocki, S. P., and Gayley, K. G., "The Prototype Colliding-Wind Pinwheel WR 104," ApJ 675, 698-710 (Mar. 2008). 
[16] Macintosh, B., Graham, J., Palmer, D., Doyon, R., Gavel, D., Larkin, J., Oppenheimer, B., Saddlemyer, L., Wallace, J. K., Bauman, B., Evans, J., Erikson, D., Morzinski, K., Phillion, D., Poyneer, L., Sivaramakrishnan, A., Soummer, R., Thibault, S., and Veran, J.-P., "The Gemini Planet Imager," in [SPIE Conference Series], 6272 (July 2006).

[17] Beuzit, J.-L., Feldt, M., Dohlen, K., Mouillet, D., Puget, P., Wildi, F., Abe, L., Antichi, J., Baruffolo, A., Baudoz, P., Boccaletti, A., Carbillet, M., Charton, J., Claudi, R., Downing, M., Fabron, C., Feautrier, P., Fedrigo, E., Fusco, T., Gach, J.-L., Gratton, R., Henning, T., Hubin, N., Joos, F., Kasper, M., Langlois, M., Lenzen, R., Moutou, C., Pavlov, A., Petit, C., Pragt, J., Rabou, P., Rigal, F., Roelfsema, R., Rousset, G., Saisse, M., Schmid, H.-M., Stadler, E., Thalmann, C., Turatto, M., Udry, S., Vakili, F., and Waters, R., "SPHERE: a planet finder instrument for the VLT," in [Society of Photo-Optical Instrumentation Engineers (SPIE) Conference Series], Society of Photo-Optical Instrumentation Engineers (SPIE) Conference Series 7014 (Aug. 2008).

[18] Sivaramakrishnan, A., Koresko, C. D., Makidon, R. B., Berkefeld, T., and Kuchner, M. J., "Ground-based Coronagraphy with High-order Adaptive Optics," ApJ 552, 397-408 (May 2001).

[19] Marois, C., Lafrenière, D., Doyon, R., Macintosh, B., and Nadeau, D., "Angular Differential Imaging: A Powerful High-Contrast Imaging Technique," ApJ 641, 556-564 (Apr. 2006).

[20] Lafrenière, D., Doyon, R., Nadeau, D., Artigau, É., Marois, C., and Beaulieu, M., "Improving the Speckle Noise Attenuation of Simultaneous Spectral Differential Imaging with a Focal Plane Holographic Diffuser," ApJ 661, 1208-1217 (June 2007).

[21] Doyon, R., Rowlands, N., Hutchings, J., Evans, C. E., Greenberg, E., Scott, A. D., Touhari, D., Beaulieu, M., Abraham, R., Ferrarese, L., Fullerton, A. W., Jayawardhana, R., Johnston, D., Meyer, M. R., Pipher, J., and Sawicki, M., "The JWST tunable filter imager (TFI)," in [Society of Photo-Optical Instrumentation Engineers (SPIE) Conference Series], Presented at the Society of Photo-Optical Instrumentation Engineers (SPIE) Conference $\mathbf{7 0 1 0}$ (Aug. 2008).

[22] Gardner, J. P., Mather, J. C., Clampin, M., Doyon, R., Greenhouse, M. A., Hammel, H. B., Hutchings, J. B., Jakobsen, P., Lilly, S. J., Long, K. S., Lunine, J. I., McCaughrean, M. J., Mountain, M., Nella, J., Rieke, G. H., Rieke, M. J., Rix, H.-W., Smith, E. P., Sonneborn, G., Stiavelli, M., Stockman, H. S., Windhorst, R. A., and Wright, G. S., "The James Webb Space Telescope," Space Science Reviews 123, 485-606 (Apr. 2006).

[23] Baudoz, P., Boccaletti, A., Riaud, P., Cavarroc, C., Baudrand, J., Reess, J. M., and Rouan, D., "Feasibility of the Four-Quadrant Phase Mask in the Mid-Infrared on the James Webb Space Telescope," PASP 118, 765-773 (May 2006).

[24] Lloyd, J. P., Gavel, D. T., Graham, J. R., Hodge, P. E., Sivaramakrishnan, A., and Voit, G. M., "Fourquadrant phase mask coronagraph: analytical calculation and pupil geometry," in [SPIE Conference Series], Schultz, A. B., ed., 4860, 171-181 (Feb. 2003).

[25] Sivaramakrishnan, A., "Observing Proposals for Coarse Phasing JWST using Dispersed Hartmann Sensing (JWST-STScI-000957)," STScI, Baltimore (2005).

[26] Jennison, R. C., "A phase sensitive interferometer technique for the measurement of the Fourier transforms of spatial brightness distributions of small angular extent," MNRAS 118, 276-+ (1958).

[27] Baldwin, J. E., Haniff, C. A., Mackay, C. D., and Warner, P. J., "Closure phase in high-resolution optical imaging," Nature 320, 595-597 (Apr. 1986).

[28] Haniff, C. A., Buscher, D. F., Christou, J. C., and Ridgway, S. T., "Synthetic aperture imaging at infrared wavelengths," MNRAS 241, 51P-56P (Nov. 1989).

[29] Readhead, A. C. S., Nakajima, T. S., Pearson, T. J., Neugebauer, G., Oke, J. B., and Sargent, W. L. W., "Diffraction-limited imaging with ground-based optical telescopes," AJ 95, 1278-1296 (Apr. 1988).

[30] Monnier, J. D., "Optical interferometry in astronomy," Reports on Progress in Physics 66, 789-857 (May 2003).

[31] Racine, R. ., Walker, G. A. H., Nadeau, D., Doyon, R. ., and Marois, C., "Speckle Noise and the Detection of Faint Companions," PASP 111, 587-594 (May 1999).

[32] Sivaramakrishnan, A., Lloyd, J. P., Hodge, P. E., and Macintosh, B. A., "Speckle Decorrelation and Dynamic Range in Speckle Noise-limited Imaging," ApJL 581, L59-L62 (Dec. 2002). 
[33] Soummer, R., Ferrari, A., Aime, C., and Jolissaint, L., "Speckle Noise and Dynamic Range in Coronagraphic Images," ApJ 669, 642-656 (Nov. 2007).

[34] Sivaramakrishnan, A., Soummer, R., Pueyo, L., Wallace, J. K., and Shao, M., "Sensing Phase Aberrations behind Lyot Coronagraphs," ApJ 688, 701-708 (Nov. 2008).

[35] Baraffe, I., Chabrier, G., Barman, T. S., Allard, F., and Hauschildt, P. H., "Evolutionary models for cool brown dwarfs and extrasolar giant planets. The case of HD 209458," Astronomy and Astrophysics 402, 701-712 (May 2003).

[36] Marley, M. S., Fortney, J. J., Hubickyj, O., Bodenheimer, P., and Lissauer, J. J., "On the Luminosity of Young Jupiters," ApJ 655, 541-549 (Jan. 2007).

[37] Krist, J. E., Beichman, C. A., Trauger, J. T., Rieke, M. J., Somerstein, S., Green, J. J., Horner, S. D., Stansberry, J. A., Shi, F., Meyer, M. R., Stapelfeldt, K. R., and Roellig, T. L., "Hunting planets and observing disks with the JWST NIRCam coronagraph," in [SPIE Conference Series], 6693 (Sept. 2007).

[38] Cavarroc, C., Boccaletti, A., Baudoz, P., Amiaux, J., and Regan, M., "Target Acquisition for MIRI Coronagraphs," PASP 120, 1016-1027 (Sept. 2008).

[39] Lightsey, P. A., Barto, A. A., and Contreras, J., "Optical performance for the James Webb Space Telescope," in [SPIE Conference Series], Mather, J. C., ed., 5487, 825-832 (Oct. 2004).

[40] Barto, A. A. and Lightsey, P. A., "Optical performance modeling of the James Webb Space Telescope," in [Optical, Infrared, and Millimeter Space Telescopes.], Mather, J. C., ed., Proc. SPIE 5487, 867-874 (Oct. 2004).

[41] Cox, C. and Hodge, P., "Point-spread function modeling for the James Webb Space Telescope," in [Space Telescopes and Instrumentation I: Optical, Infrared, and Millimeter.], John C. Mather, Howard A. MacEwen, and Mattheus W. M. de Graauw, eds., Proc. SPIE 6265, 62650W (2006).

[42] Sivaramakrishnan, A., Makidon, R. B., Figer, D. F., Jedrzejewski, R. I., Bushouse, H. A., Krist, J. E., Stockman, H. S. P., Hodge, P., Dencheva, N. M., Rauscher, B. J., Laidler, V. G., Ohara, C. M., Redding, D. C., Im, M., and Offenberg, J. D., "NIRCAM image simulations for NGST wavefront sensing," in [IR Space Telescopes and Instruments.], John C. Mather, ed., Proc. SPIE 4850, 388-397 (2003).

[43] Sivaramakrishnan, A., Morse, E. C., Makidon, R. B., Bergeron, L. E., Casertano, S., Figer, D. F., Acton, D. S., Atcheson, P. D., and Rieke, M. J., "Limits on routine wavefront sensing with NIRCam on JWST," in [SPIE Conference Series], Mather, J. C., ed., 5487, 909-917 (Oct. 2004).

[44] Soummer, R., Pueyo, L., Sivaramakrishnan, A., and Vanderbei, R. J., "Fast computation of Lyot-style coronagraph propagation," Optics Express 15, 15935-+ (2007).

[45] Makidon, R. B., Sivaramakrishnan, A., Soummer, R., Anderson, J., and van der Marel, R. P., "Towards observing extrasolar giant-planet environments with JWST," in [SPIE Conference Series], 7010 (Aug. 2008).

[46] Kraus, A. L., Ireland, M. J., Martinache, F., and Lloyd, J. P., "Mapping the Shores of the Brown Dwarf Desert. I. Upper Scorpius," ApJ 679, 762-782 (May 2008).

[47] Koekemor, A. M. and Lindsay, K., "An investigation of optimal dither strategies for JWST (JWST-STScI000674 SM-12)," STScI, Baltimore (2005).

[48] Sivaramakrishnan, A., "Coarse Phasing JWST: an Operations Concept Document (JWST-STScI-000378)," STScI, Baltimore (2004).

[49] Sivaramakrishnan, A., Makidon, R. B., Acton, D. S., and Shi, F., "Coarse Phasing JWST using Dispersed Fringe Sensing and Dispersed Hartmann Sensing during Commissioning (JWST-STScI-000341)," STScI, Baltimore (2005).

[50] Long, K. S., "JWST Mission Operations Concept Document (JWST-OPS-002018)," STScI, Baltimore (2006). Revision B. 